\title{
NUMERICAL ANALYSIS OF STOCHASTIC SCHEMES IN GEOPHYSICS*
}

\author{
BRIAN D. EWALD ${ }^{\dagger}$ AND ROGER TÉMAM TE $^{\ddagger}$
}

\begin{abstract}
We present and study the stability, convergence, and order of convergence of a numerical scheme used in geophysics, namely, the stochastic version of a deterministic "implicit leapfrog" scheme which has been developed for the approximation of the so-called barotropic vorticity model. Two other schemes which might be useful in the context of geophysical applications are also introduced and discussed.
\end{abstract}

Key words. numerical methods, stochastic differential equations, leapfrog scheme, AdamsBashforth scheme, geophysical fluid dynamics

AMS subject classifications. 65C $30,60 \mathrm{H} 35,65 \mathrm{~N} 12,86 \mathrm{~A} 10$

DOI. $10.1137 /$ S0036142902418333

1. Introduction. Much effort has been invested in studying numerical schemes for stochastic differential equations of the form

$$
d U_{t}=a\left(U_{t}\right) d t+b\left(U_{t}\right) d W_{t}
$$

where $U_{t} \in \mathbb{R}^{d}, a$ is a function from $\mathbb{R}^{d}$ into itself, $W$ is a Wiener process on $\mathbb{R}^{m}$, and $b$ is a function from $\mathbb{R}^{d}$ into $\mathbb{R}^{d \times m}$.

For the so-called weak approximation of (1.1), in which the approximation of the expectation of functions of $U$ is considered, extensive work is due, for example, to Talay and his collaborators, work relying on probabilistic methods more involved than those used in this article (see, e.g., [1], [2], [12] and the references therein).

The question of strong approximation of (1.1), in which the approximation of sample paths of $U$ is desired, has also been much studied. Mil'shtein, in [8], introduced the scheme

$$
\begin{aligned}
U_{n+1}^{k}= & U_{n}^{k}+\sum_{j=1}^{d} b^{k, j}\left(U_{n}\right) \Delta W_{n}^{j}+a^{k}\left(U_{n}\right) \Delta t \\
& +\sum_{j_{1}, j_{2}, \ell=1}^{d} b^{\ell, j_{1}}\left(U_{n}\right) \frac{\partial b^{k, j_{2}}}{\partial x^{\ell}}\left(U_{n}\right) \int_{t_{n}}^{t_{n+1}}\left(W_{s}^{j_{1}}-W_{t_{n}}^{j_{1}}\right) d W_{s}^{j_{2}}
\end{aligned}
$$

which converges to $U$ to the order of $\Delta t$ in mean-square error. His method involved the consideration of a functional analytic Taylor series for the infinitesimal generator of a semigroup corresponding to $U$ and $W$. Rümelin later investigated a stochastic analogue of Runge-Kutta (RK) schemes in [10], in which he compared them to Mil'shtein's scheme. The RK schemes which he derives can be arranged to converge

*Received by the editors November 20, 2002; accepted for publication (in revised form) September 25, 2003; published electronically March 31, 2005. This work was supported in part by a grant from the National Science Foundation (NSF-DMS 0074334) and by the Research Fund of Indiana University.

http://www.siam.org/journals/sinum/42-6/41833.html

$\dagger$ Department of Mathematics, Texas A\&M University, College Station, TX 77843 (ewald@math. tamu.edu).

${ }^{\ddagger}$ Department of Mathematics, Institute for Scientific Computing and Applied Mathematics, Indiana University, Bloomington, IN 47405 (temam@indiana.edu). 
to $U$ when the stochastic integral is interpreted in the sense of Itô, Stratonovich, or in fact for any stochastic calculus whatsoever. However, the issue of the accuracy of these RK schemes (which is not the same as in the deterministic case) is not fully addressed in $[10]$ and is mostly unresolved. In fact, there are indications that these stochastic RK schemes are of significantly lower orders of accuracy than their deterministic counterparts (see [3]).

The book by Kloeden and Platen [6] and the companion volume by Kloeden, Platen, and Schurz [7] offer a systematic investigation of numerical schemes for (1.1) in both the sense of Itô and of Stratonovich, the two stochastic calculi which in applications are by far the most useful. Their methods are analytic and are applicable to proving the convergence of a wide range of numerical schemes, and they derive a very general scheme (formula (12.6.2) of [6]) which, for various choices of parameters, includes stochastic analogues of such deterministic schemes as the explicit and implicit Euler schemes, the Crank-Nicholson scheme, and the leapfrog scheme.

In the geophysics community, an enormous amount of work has been spent in developing large, complex numerical models of the oceans and atmosphere. The questions therefore arise: Is it possible to add stochastic numerical noise to these already existing models in such a way that it is known to what the scheme converges (e.g., to the Itô or Stratonovich solution of some stochastic differential equation), to what order they may be expected to converge, etc.? While we certainly do not answer these complex questions here, we consider a simple "implicit leapfrog" scheme for a barotropic model (supplied to us by Cecile Penland and Prashant Sardeshmukh) and demonstrate one way of adding stochastic noise to it so that these questions can be answered for the resulting stochastic scheme (section 4).

This scheme and the scheme in section 5 have been applied in investigating El Niño (see [4]). In this paper, the schemes were used for the numerical timestepping to determine if a linear inverse model of El Niño (see [9]) can be reconciled with the observed skew toward warm events in the Pacific. It was found that the observed skew is well within the range predicted by the model, although the observed trend is not.

We also propose a stochastic analogue for the deterministic Adams-Bashforth scheme, using methods similar to those of [6], as an attempt to produce alternate schemes which are higher order in time (studied in section 3, following the preliminary results in section 2).

Last, we examine the derivatives of $a$ and $b$ which occur naturally in the above schemes, and which can prove to be troublesome in certain applications in which these functions, especially $b$, are given by physical parametrizations (i.e., by "tables") and not by analytic expressions. We consider how these derivatives can be replaced by finite differences derived from space-discretization while still maintaining the existing rate of convergence (section 5).

We realize that the results of this article, while very useful in our opinion, are just some small contribution to an outstanding problem, namely, the numerical analysis of stochastic differential equations which raise - with more difficulty - the same issues as in the deterministic case: consistency, convergence, and accuracy. All of these issues - partly due to the form of the stochastic Taylor formula - are considerably more difficult than in the deterministic case; in particular, consistency includes here the issue of the type of stochastic calculus (Itô, Stratonovich, or otherwise) to which the scheme converges.

In the case of the geoscience scheme, the scheme that we study in section 4 is the closest we could get, at this time, to a scheme actually used in the geo- 
sciences, without any prior information on its consistency, convergence, and accuracy properties. The version of the Adams-Bashforth scheme studied in section 3 has given very good numerical results in simulations for simple (one-dimensional) stochastic differential equations; see section 6 and forthcoming articles. The numerical example in section 6 is actually based on a mistake: one of the stochastic processes involved in the scheme was mistakenly believed to be (and treated as) a Gaussian process; this did not affect the accuracy of the scheme, which remains at order two as predicted by the theory. This raises perhaps an interesting probabilistic problem about the approximation of certain non-Gaussian processes used in numerical schemes. Another issue of probabilistic nature is raised by the Adams-Bashforth-type scheme studied in section 3: in several or in high dimensions, a large number of stochastic processes need to be simulated, which could make the cost prohibitive. It is not excluded that future probabilistic developments will improve this situation. In particular, the first author, using some ideas of Gaines and Lyons [5], is trying at this time to develop methods of generating the needed stochastic increments.

As we have said, there are, of course, a great many mathematical difficulties which this paper does not address. However, methods involving stochastic noise are already in common use in numerical simulations for the geosciences and turbulence (and, no doubt, many other areas in science). As mathematicians, we can attempt to help these scientists develop the necessary numerical tools, or watch as they do it themselves.

2. Preliminary results. We consider a stochastic differential equation

$$
d U_{t}=a\left(t, U_{t}\right) d t+b\left(t, U_{t}\right) d W_{t}
$$

for $U=\left(u_{1}, \ldots, u_{d}\right) \in \mathbb{R}^{d}$, where $a: \mathbb{R}^{+} \times \mathbb{R}^{d} \rightarrow \mathbb{R}^{d}, b: \mathbb{R}^{+} \times \mathbb{R}^{d} \rightarrow \mathbb{R}^{d \times m}$, and $W$ is a Wiener process in $\mathbb{R}^{m}$ adapted to a filtration $\left\{\mathcal{F}_{t}\right\}_{t \geq 0}$.

We then have the Itô formula, which states that if $F: \mathbb{R}^{+} \times \mathbb{R}^{d} \rightarrow \mathbb{R}^{\hat{d}}$, then $F_{t}=F\left(t, U_{t}\right)$ satisfies the stochastic differential equation

$$
d F_{t}=\left[\frac{\partial F}{\partial t}+a^{k}\left(F_{t}\right) \frac{\partial F}{\partial u^{k}}+\frac{1}{2} b^{i j}\left(F_{t}\right) b^{k j}\left(F_{t}\right) \frac{\partial^{2} F}{\partial u^{i} \partial u^{k}}\right] d t+b^{i j}\left(F_{t}\right) \frac{\partial F}{\partial u^{i}} d W_{t}^{j} ;
$$

here we use the Einstein convention for repeated indices.

We use the following notation from [6]: We call a row vector $\alpha=\left(j_{1}, j_{2}, \ldots, j_{l}\right)$, where each $j_{i} \in\{0,1, \ldots, m\}$, a multi-index of length $l=\ell(\alpha) \in\{1,2, \ldots\}$. We also use $\nu$ to denote the multi-index of length 0 , i.e., $\ell(\nu)=0$. We define $n(\alpha)$ to be the number of entries of $\alpha$ which are 0 . For adapted, right-continuous functions $f$, and stopping times $\rho, \tau$ such that $0 \leq \rho \leq \tau \leq T$ almost surely, we define

$$
I_{\alpha}[f(\cdot)]_{\rho, \tau}= \begin{cases}f(\tau) & \text { if } \ell(\alpha)=0, \\ \int_{\rho}^{\tau} I_{\alpha-}[f(\cdot)]_{\rho, s} d s & \text { if } \ell(\alpha) \geq 1, j_{\ell(\alpha)}=0, \\ \int_{p}^{\tau} I_{\alpha-}[f(\cdot)]_{\rho, s} d W_{s}^{j_{\ell(\alpha)}} & \text { if } \ell(\alpha) \geq 1, j_{\ell(\alpha)} \neq 0 .\end{cases}
$$

Here $\alpha-$ is $\alpha$ with its final component removed.

We define the spaces $\mathcal{H}_{\alpha}$ as follows.

First, $\mathcal{H}_{\nu}$ is the space of adapted right-continuous stochastic processes $f$ with left limits such that $|f(t)|$ is almost surely finite for each $t \geq 0$. Next, $\mathcal{H}_{(0)}$ contains those elements of $\mathcal{H}_{\nu}$ such that

$$
\int_{0}^{t}|f(s)| d s<\infty
$$


almost surely for each $t \geq 0$; and $\mathcal{H}_{(j)}$ for $j \neq 0$ contains those elements of $\mathcal{H}_{\nu}$ such that

$$
\int_{0}^{t}|f(s)|^{2} d s<\infty
$$

almost surely for each $t \geq 0$. Finally, if $\ell(\alpha) \geq 2$, we define $\mathcal{H}_{\alpha}$ recursively as those elements of $\mathcal{H}_{\nu}$ that satisfy

$$
I_{\alpha-}[f(\cdot)]_{0, t} \in \mathcal{H}_{\left(j_{\ell(\alpha)}\right)}
$$

almost surely for all $t \geq 0$.

We also define the operators

$$
\begin{gathered}
L^{0}=\frac{\partial}{\partial t}+a^{k} \frac{\partial}{\partial u^{k}}+\frac{1}{2} b^{k j} b^{l j} \frac{\partial^{2}}{\partial u^{k} \partial u^{l}}, \\
L^{j}=b^{k j} \frac{\partial}{\partial u^{k}}
\end{gathered}
$$

and, if $f \in C^{h}\left(\mathbb{R}^{+} \times \mathbb{R}^{d}, \mathbb{R}\right)$, where $h \geq \ell(\alpha)+n(\alpha)$, we set

$$
f_{\alpha}= \begin{cases}f & \text { if } \ell(\alpha)=0, \\ L^{j_{1}} f_{-\alpha} & \text { if } \ell(\alpha) \geq 1 .\end{cases}
$$

Here $-\alpha$ is $\alpha$ with its first component removed.

We note that if $f(t, u) \equiv u$, then $f_{(0)}=a, f_{(j)}=b^{j}$, etc. In what follows, unless explicitly stated otherwise, we will assume that $f$ is this identity function.

A set, $\mathcal{A}$, of multi-indices is said to be a hierarchical set if $\mathcal{A} \neq \emptyset, \sup _{\alpha \in \mathcal{A}} \ell(\alpha)<$ $\infty$, and $-\alpha \in \mathcal{A}$ whenever $\alpha \in \mathcal{A}-\{\nu\}$. We then define the remainder set $\mathcal{B}(\mathcal{A})$ of $\mathcal{A}$ by $\mathcal{B}(\mathcal{A})=\{\alpha \mid \alpha \notin \mathcal{A}$ and $-\alpha \in \mathcal{A}\}$. We can now provide a stochastic Taylor expansion for $U$ satisfying (2.1): If $f: \mathbb{R}^{+} \times \mathbb{R}^{d} \rightarrow \mathbb{R}$, then, provided the derivatives and integrals exist,

$$
f\left(\tau, U_{\tau}\right)=\sum_{\alpha \in \mathcal{A}} I_{\alpha}\left[f_{\alpha}\left(\rho, U_{\rho}\right)\right]_{\rho, \tau}+\sum_{\alpha \in \mathcal{B}(\mathcal{A})} I_{\alpha}\left[f_{\alpha}(\cdot, U .)\right]_{\rho, \tau},
$$

where $\mathcal{A}$ is some hierarchical set.

Now, for $\gamma=0.5,1.0,1.5, \ldots$, we set

$$
\mathcal{A}_{\gamma}=\left\{\alpha \mid \ell(\alpha)+n(\alpha) \leq 2 \gamma \text { or } \ell(\alpha)=n(\alpha)=\gamma+\frac{1}{2}\right\} .
$$

We call the stochastic Taylor expansion with $\mathcal{A}=\mathcal{A}_{\gamma}$ the stochastic Taylor expansion to order $\gamma$.

We will make use of the following lemmas in the succeeding sections. In each of them, $U$ is the solution to (2.1), and $t_{k}=k \Delta t$ for $k=0,1, \ldots, N$ is an equipartition of $[0, T]$, so that $t_{N}=T$; we partly rely on [6] for the proofs.

Lemma 2.1. Suppose $Y_{n}$ is a stochastic process adapted to the filtration $\mathcal{F}_{t}$ at the equipartition (i.e., $Y_{n}$ is $\mathcal{F}_{t_{n}}$-measurable), the function $f$ satisfies $|f(t, x)-f(t, y)|$ $\leq K|x-y|$ for all $t \in[0, T]$ and $x, y \in \mathbb{R}$, and $\alpha$ is a multi-index with $\ell(\alpha) \geq 1$. Then

$$
\begin{gathered}
\mathbb{E} \sup _{0 \leq m \leq n}\left|\sum_{k=0}^{m-1} I_{\alpha}\left[f\left(t_{k}, U_{t_{k}}\right)-f\left(t_{k}, Y_{k}\right)\right]_{t_{k}, t_{k+1}}\right|^{2} \\
\leq C \Delta t \sum_{k=0}^{n-1} \mathbb{E} \sup _{0 \leq m \leq k}\left|U_{t_{m}}-Y_{m}\right|^{2}
\end{gathered}
$$


Proof. For $\alpha=(0)$, we have

$$
\begin{aligned}
& \mathbb{E} \sup _{0 \leq m \leq n}\left|\sum_{k=0}^{m-1}\left(f\left(t_{k}, U_{t_{k}}\right)-f\left(t_{k}, Y_{k}\right)\right) \Delta t\right|^{2} \\
& \leq \Delta t^{2} \mathbb{E} \sup _{0 \leq m \leq n} m \sum_{k=0}^{m-1}\left|f\left(t_{k}, U_{t_{k}}\right)-f\left(t_{k}, Y_{k}\right)\right|^{2} \\
& \leq n \Delta t^{2} \mathbb{E} \sum_{k=0}^{n-1} K^{2}\left|U_{t_{k}}-Y_{k}\right|^{2} \\
& \leq K^{2} T \Delta t \sum_{k=0}^{n-1} \mathbb{E} \sup _{0 \leq m \leq k}\left|U_{t_{m}}-Y_{m}\right|^{2} .
\end{aligned}
$$

For $\alpha=(j)$,

$$
\begin{aligned}
\mathbb{E} \sup _{0 \leq m \leq n}\left|\sum_{k=0}^{m-1}\left(f\left(t_{k}, U_{t_{k}}\right)-f\left(t_{k}, Y_{k}\right)\right) \Delta W_{k}^{j}\right|^{2} \\
\leq 4 \mathbb{E}\left|\sum_{k=0}^{n-1}\left(f\left(t_{k}, U_{t_{k}}\right)-f\left(t_{k}, Y_{k}\right)\right) \Delta W_{k}^{j}\right|^{2} \\
\leq 4 \sum_{k=0}^{n-1} \mathbb{E}\left|f\left(t_{k}, U_{t_{k}}\right)-f\left(t_{k}, Y_{k}\right)\right|^{2} \Delta t \\
\leq 4 K^{2} \Delta t \sum_{k=0}^{n-1} \mathbb{E} \sup _{0 \leq m \leq k}\left|U_{t_{k}}-Y_{k}\right|^{2} .
\end{aligned}
$$

For longer $\alpha$ 's, we just repeat the above two arguments as necessary.

Lemma 2.2. Suppose the function $f$ satisfies $|f(t, x)|^{2} \leq K^{2}\left(1+|x|^{2}\right)$ for all $t \in[0, T]$ and $x \in \mathbb{R}$, and that $\alpha$ is a multi-index with $\ell(\alpha) \geq 1$. Then

$$
\mathbb{E} \sup _{0 \leq m \leq n}\left|\sum_{k=0}^{m-1} I_{\alpha}[f(\cdot, U .)]_{t_{k}, t_{k+1}}\right|^{2} \leq \begin{cases}C \Delta t^{2(\ell(\alpha)-1)}\left(1+\mathbb{E}\left|U_{0}\right|^{2}\right) & \text { if } \ell(\alpha)=n(\alpha), \\ C \Delta t^{\ell(\alpha)+n(\alpha)-1}\left(1+\mathbb{E}\left|U_{0}\right|^{2}\right) & \text { if } \ell(\alpha) \neq n(\alpha) .\end{cases}
$$

Proof. The ideas of this proof are the same as those in the proof of Lemma 2.1, along with the following bound on the solution $U_{t}$ (see equation (4.5.16) of [6]):

$$
\mathbb{E} \sup _{t_{0} \leq s \leq T}\left|U_{s}\right|^{2} \leq C\left(1+\mathbb{E}\left|U_{t_{0}}\right|^{2}\right) .
$$

If we apply Lemma 10.8.1 of [6] with $g(s)=f\left(s, U_{s}\right)$, we have

$$
\begin{aligned}
\mathbb{E} \sup _{0 \leq m \leq n}\left|\sum_{k=0}^{m-1} I_{\alpha}[f(\cdot, U .)]_{t_{k}, t_{k+1}}\right|^{2} \\
\quad \leq \begin{cases}C \Delta t^{2(\ell(\alpha)-1)} \int_{t_{0}}^{t_{n}} \mathbb{E} \sup _{t_{0} \leq s \leq t}\left|f\left(s, U_{s}\right)\right|^{2} d t & \text { if } \ell(\alpha)=n(\alpha), \\
C \Delta t^{\ell(\alpha)+n(\alpha)-1} \int_{t_{0}}^{t_{n}} \mathbb{E} \sup _{t_{0} \leq s \leq t}\left|f\left(s, U_{s}\right)\right|^{2} d t & \text { if } \ell(\alpha) \neq n(\alpha) .\end{cases}
\end{aligned}
$$


Here, the constant $C$ depends only on the length of the time interval $T-t_{0}$ and on $\alpha$.

We then apply (2.16) and the growth condition on $f$, and we have the desired result.

LEMma 2.3. Suppose that the sequence of positive numbers $Z_{n}$ for $n=0,1, \ldots, N$ satisfies the inequality

$$
Z_{n} \leq C\left(\Delta t \sum_{k=0}^{n} Z_{k}+\Delta t^{\gamma}\right)
$$

for some positive constant $C$ and some $\gamma>0$. Then $Z_{N}=O\left(\Delta t^{\gamma}\right)$ as $\Delta t \rightarrow 0$.

Proof. Set $\xi_{n}=\Delta t \sum_{k=0}^{n} Z_{k}$, so $Z_{n}=\frac{1}{\Delta t}\left(\xi_{n}-\xi_{n-1}\right)$, and we have

$$
\frac{1}{\Delta t}\left(\xi_{n}-\xi_{n-1}\right) \leq C \xi_{n}+C \Delta t^{\gamma} .
$$

That is,

$$
(1-C \Delta t) \xi_{n} \leq \xi_{n-1}+C \Delta t^{\gamma+1} .
$$

Therefore,

$$
\begin{aligned}
(1-C \Delta t)^{n} \xi_{n} & \leq(1-C \Delta t)^{n-1} \xi_{n-1}+(1-C \Delta t)^{n-1} C \Delta t^{\gamma+1}, \\
(1-C \Delta t)^{n-1} \xi_{n-1} \leq & (1-C \Delta t)^{n-2} \xi_{n-2}+(1-C \Delta t)^{n-2} C \Delta t^{\gamma+1}, \\
& \vdots \\
(1-C \Delta t) \xi_{1} & \leq C \Delta t^{\gamma+1},
\end{aligned}
$$

and, summing,

$$
\begin{aligned}
(1-C \Delta t)^{n} \xi_{n} & \left.\leq\left(1+(1-C \Delta t)+\cdots+(1-C \Delta t)^{n-1}\right)\right) C \Delta t^{\gamma+1} \\
& \leq \frac{1-(1-C \Delta t)^{n}}{1-(1-C \Delta t)} C \Delta t^{\gamma+1} \\
& \leq\left(1-(1-C \Delta t)^{n}\right) \Delta t^{\gamma} .
\end{aligned}
$$

Since $(1-C \Delta t)^{N} \rightarrow e^{-C T}$ as $N=T / \Delta t \rightarrow \infty$, we see that $\xi_{n} \leq C \Delta t^{\gamma}$ for some (different) $C$. Thus, by (2.18),

$$
Z_{n} \leq C\left(\xi_{n}+\Delta t^{\gamma}\right) \leq C \Delta t^{\gamma} .
$$

3. A stochastic Adams-Bashforth scheme. The deterministic Adams-Bashforth scheme for the ordinary differential equation $\phi^{\prime}=F(\phi)$ takes the form

$$
\phi_{n+1}=\phi_{n}+\frac{\Delta t}{2}\left[3 F\left(\phi_{n}\right)-F\left(\phi_{n-1}\right)\right] .
$$

This scheme is order $\Delta t^{2}$. We will derive a stochastic version of this scheme which maintains the same order.

We begin with the stochastic Taylor expansion to order $\gamma=2.0$ :

$$
\begin{aligned}
U_{t+\Delta}= & U_{t}+b^{j} \Delta W^{j}+a \Delta+L^{j_{1}} b^{j_{2}} I_{\left(j_{1}, j_{2}\right)}+L^{0} b^{j} I_{(0, j)}+L^{j} a I_{(j, 0)} \\
& +L^{j_{1}} L^{j_{2}} b^{j_{3}} I_{\left(j_{1}, j_{2}, j_{3}\right)}+\frac{1}{2} L^{0} a \Delta^{2}+L^{0} L^{j_{1}} b^{j_{2}} I_{\left(0, j_{1}, j_{2}\right)}+L^{j_{1}} L^{0} b^{j_{2}} I_{\left(j_{1}, 0, j_{2}\right)} \\
& +L^{j_{1}} L^{j_{2}} a I_{\left(j_{1}, j_{2}, 0\right)}+L^{j_{1}} L^{j_{2}} L^{j_{3}} b^{j_{4}} I_{\left(j_{1}, j_{2}, j_{3}, j_{4}\right)}+\tilde{R}_{2.0}^{\Delta}(t) \\
= & U_{t}+a \Delta+\frac{1}{2} L^{0} a \Delta^{2}+M^{\Delta}(t)
\end{aligned}
$$


where each coefficient is at the point $\left(t, U_{t}\right)$, and each stochastic integral is from $t$ to $t+\Delta, \Delta=\Delta t$. We have also used the Einstein summation convention.

Similarly, for $\gamma=1.5$, we have

$$
a\left(t+\Delta, U_{t+\Delta}\right)=a+L^{0} a \Delta+N^{\Delta}(t),
$$

where $N^{\Delta}(t)=\frac{1}{2} L^{0} L^{0} a \Delta^{2}+L^{j} a \Delta W^{j}+L^{0} L^{j} a I_{(0, j)}+L^{j} L^{0} a I_{(j, 0)}+L^{j_{1}} L^{j_{2}} a I_{\left(j_{1}, j_{2}\right)}+$ $L^{j_{1}} L^{j_{2}} L^{j_{3}} a I_{\left(j_{1}, j_{2}, j_{3}\right)}+\tilde{R}_{1.5}^{\Delta}(t)$, and, for $\gamma=1.0$,

$$
L^{0} a\left(t+\Delta, U_{t+\Delta}\right)=L^{0} a+P^{\Delta}(t),
$$

where $P^{\Delta}(t)=L^{0} L^{0} a \Delta+L^{j} L^{0} a \Delta W^{j}+L^{j_{1}} L^{j_{2}} L^{0} a I_{\left(j_{1}, j_{2}\right)}+\tilde{R}_{1.0}^{\Delta}(t)$.

Combining these results, we get

$$
\begin{aligned}
U_{t+\Delta}= & U_{t}+\left[\alpha a\left(t+\Delta, U_{t+\Delta}\right)+(1-\alpha) a\right] \Delta \\
& +\left(\frac{1}{2}-\alpha\right)\left[\beta L^{0} a\left(t+\Delta, U_{t+\Delta}\right)+(1-\beta) L^{0} a\right] \Delta^{2} \\
& -\alpha \Delta N^{\Delta}(t)-\left(\frac{1}{2}-\alpha\right) \beta \Delta^{2} P^{\Delta}(t)+M^{\Delta}(t) .
\end{aligned}
$$

In particular, if $t=t_{n}, \Delta=2 \Delta t, \alpha=0, \beta=0$, and writing $U_{n}=U_{t_{n}}$,

$$
U_{n+2}=U_{n}+2 a\left(t_{n}, U_{n}\right) \Delta t+2 L^{0} a\left(t_{n}, U_{n}\right) \Delta t^{2}+M^{2 \Delta t}\left(t_{n}\right)
$$

and if $t=t_{n}, \Delta=\Delta t, \alpha=-\frac{3}{2}, \beta=0$,

$$
\begin{aligned}
U_{n+1}= & U_{n}-\frac{3}{2} a\left(t_{n+1}, U_{n+1}\right) \Delta t+\frac{5}{2} a\left(t_{n}, U_{n}\right) \Delta t \\
& +2 L^{0} a\left(t_{n}, U_{n}\right) \Delta t^{2}+\frac{3}{2} N^{\Delta t}\left(t_{n}\right) \Delta t+M^{\Delta t}\left(t_{n}\right) .
\end{aligned}
$$

Therefore,

$$
\begin{aligned}
U_{n+2}= & U_{n+1}+\left(U_{n+2}-U_{n}\right)-\left(U_{n+1}-U_{n}\right) \\
= & U_{n+1}+\left[\frac{3}{2} a\left(t_{n+1}, U_{n+1}\right)-\frac{1}{2} a\left(t_{n}, U_{n}\right)\right] \Delta t \\
& -\frac{3}{2} \Delta t N^{\Delta t}\left(t_{n}\right)+\left(M^{2 \Delta t}\left(t_{n}\right)-M^{\Delta t}\left(t_{n}\right)\right) .
\end{aligned}
$$

This leads us to consider the following stochastic Adams-Bashforth (SAB) scheme:

$$
\begin{aligned}
Y_{n+2}= & Y_{n+1}+\left[\frac{3}{2} a\left(t_{n+1}, Y_{n+1}\right)-\frac{1}{2} a\left(t_{n}, Y_{n}\right)\right] \Delta t \\
& -\frac{3}{2} \Delta t A_{n}\left(t_{n}, Y_{n}\right)+B_{n}\left(t_{n}, Y_{n}\right)
\end{aligned}
$$

in which

$$
A_{n}(t, x)=L^{j} a(t, x) \Delta W^{j}+L^{j_{1}} L^{j_{2}} a(t, x) I_{\left(j_{1}, j_{2}\right)},
$$


where the random intervals are from time $t_{n}$ to $t_{n+1}$, and

$$
\begin{aligned}
B_{n}(t, x)= & b^{j}(t, x) \Delta W^{j}+L^{0} b^{j}(t, x) I_{(0, j)}+L^{j} a(t, x) I_{(j, 0)} \\
& +L^{j_{1}} b^{j_{2}}(t, x) I_{\left(j_{1}, j_{2}\right)}+L^{0} L^{j_{1}} b^{j_{2}}(t, x) I_{\left(0, j_{1}, j_{2}\right)} \\
& +L^{j_{1}} L^{0} b^{j_{2}}(t, x) I_{\left(j_{1}, 0, j_{2}\right)}+L^{j_{1}} L^{j_{2}} a(t, x) I_{\left(j_{1}, j_{2}, 0\right)} \\
& +L^{j_{1}} L^{j_{2}} b^{j_{3}}(t, x) I_{\left(j_{1}, j_{2}, j_{3}\right)}+L^{j_{1}} L^{j_{2}} L^{j_{3}} b^{j_{4}}(t, x) I_{\left(j_{1}, j_{2}, j_{3}, j_{4}\right)},
\end{aligned}
$$

where the random intervals are those from time $t_{n}$ to $t_{n+2}$ minus those from time $t_{n}$ to $t_{n+1}$.

We then have the following theorem.

THEOREM 3.1. Suppose that the coefficient functions $f_{\alpha}$ satisfy

$$
\left|f_{\alpha}(t, x)-f_{\alpha}(t, y)\right| \leq K|x-y|
$$

for all $\alpha \in \mathcal{A}_{2.0}, t \in[0, T]$, and $x, y \in \mathbb{R}^{d}$;

$$
f_{-\alpha} \in C^{1,2} \text { and } f_{\alpha} \in \mathcal{H}_{\alpha}
$$

for all $\alpha \in \mathcal{A}_{2.0} \cup \mathcal{B}\left(\mathcal{A}_{2.0}\right)$; and

$$
\left|f_{\alpha}(t, x)\right| \leq K(1+|x|)
$$

for all $\alpha \in \mathcal{A}_{2.0} \cup \mathcal{B}\left(\mathcal{A}_{2.0}\right), t \in[0, T]$, and $x \in \mathbb{R}^{d}$. Choose $\Delta t \leq 1$ and set $N=T / \Delta t$, and define $t_{n}=n \Delta t$ for $n=1, \ldots, N$. Suppose that $Y_{0}$ is some (nonrandom) initial condition and that some appropriate numerical scheme is used to generate $Y_{1}$ such that $\mathbb{E}\left[\left|U_{t_{1}}-Y_{1}\right|^{2} \mid \mathcal{F}_{0}\right]^{\frac{1}{2}} \leq C \Delta t^{2}$. Then

$$
\mathbb{E}\left[\sup _{0 \leq n \leq N}\left|U_{t_{n}}-Y_{n}\right|^{2} \mid \mathcal{F}_{0}\right]^{\frac{1}{2}} \leq C \Delta t^{2} .
$$

Proof. First, we note that

$$
\begin{aligned}
U_{n+2}= & U_{n+1}+\left[\frac{3}{2} a\left(t_{n+1} U_{n+1}\right)-\frac{1}{2} a\left(t_{n}, U_{n}\right)\right] \Delta t \\
& -\frac{3}{2} \Delta t A_{n}\left(t_{n}, U_{n}\right)+B_{n}\left(t_{n}, U_{n}\right)+R_{n},
\end{aligned}
$$

where

$$
\begin{aligned}
R_{n}= & \frac{3}{2} \Delta t\left[\frac{1}{2} L^{0} L^{0} a\left(t_{n}, U_{n}\right) \Delta t^{2}+L^{0} L^{j} a\left(t_{n}, U_{n}\right) I_{(0, j)}+L^{j} L^{0} a\left(t_{n}, U_{n}\right) I_{(j, 0)}\right. \\
& \left.+L^{j_{1}} L^{j_{2}} L^{j_{3}} a\left(t_{n}, U_{n}\right) I_{\left(j_{1}, j_{2}, j_{3}\right)}+\tilde{R}_{1.5}^{\Delta t}\left(t_{n}\right)\right]+\tilde{R}_{2.0}^{2 \Delta t}\left(t_{n}\right)-\tilde{R}_{2.0}^{\Delta t}\left(t_{n}\right) .
\end{aligned}
$$


If we iterate (3.9) and (3.16), we arrive at

$$
\left\{\begin{aligned}
U_{n}= & U_{1}+\Delta t \sum_{k=0}^{n-2}\left[\frac{3}{2} a\left(t_{k+1}, U_{k+1}\right)-\frac{1}{2} a\left(t_{k}, U_{k}\right)\right] \\
& -\frac{3}{2} \Delta t \sum_{k=0}^{n-2} A_{k}\left(t_{k}, U_{k}\right)+\sum_{k=0}^{n-2} B_{k}\left(t_{k}, U_{k}\right)+\sum_{k=0}^{n-2} R_{k}, \\
Y_{n}= & Y_{1}+\Delta t \sum_{k=0}^{n-2}\left[\frac{3}{2} a\left(t_{k+1}, Y_{k+1}\right)-\frac{1}{2} a\left(t_{k}, Y_{k}\right)\right] \\
& -\frac{3}{2} \Delta t \sum_{k=0}^{n-2} A_{k}\left(t_{k}, Y_{k}\right)+\sum_{k=0}^{n-2} B_{k}\left(t_{k}, Y_{k}\right) .
\end{aligned}\right.
$$

Set $\zeta_{n}=U_{n}-Y_{n}$. Then

$$
\begin{aligned}
\zeta_{n}= & \zeta_{1}+\Delta t \sum_{k=0}^{n-2}\left[\frac{3}{2}\left(a\left(t_{k+1}, U_{k+1}\right)-a\left(t_{k+1}, Y_{k+1}\right)\right)-\frac{1}{2}\left(a\left(t_{k}, U_{k}\right)-a\left(t_{k}, Y_{k}\right)\right)\right] \\
& -\frac{3}{2} \Delta t \sum_{k=0}^{n-2}\left(A_{k}\left(t_{k}, U_{k}\right)-A_{k}\left(t_{k}, Y_{k}\right)\right) \\
& +\sum_{i=0}^{n-2}\left(B_{k}\left(t_{k}, U_{k}\right)-B_{k}\left(t_{k}, Y_{k}\right)\right)+\sum_{k=0}^{n-2} R_{k} .
\end{aligned}
$$

Set $Z_{n}=\mathbb{E}\left[\sup _{0 \leq m \leq n}\left|\zeta_{m}\right|^{2} \mid \mathcal{F}_{0}\right]$.

Then we have

$$
\begin{aligned}
Z_{n} \leq & C\left(\mathbb{E}\left[\left|\zeta_{1}\right|^{2} \mid \mathcal{F}_{0}\right]+\Delta t^{2} \mathbb{E}\left[\sup _{0 \leq m \leq n}\left|\sum_{k=0}^{m-2} a\left(t_{k+1}, U_{k+1}\right)-a\left(t_{k+1}, Y_{k+1}\right)\right|^{2} \mid \mathcal{F}_{0}\right]\right. \\
& +\Delta t^{2} \mathbb{E}\left[\sup _{0 \leq m \leq n}\left|\sum_{k=0}^{m-2} a\left(t_{k}, U_{k}\right)-a\left(t_{k}, Y_{k}\right)\right|^{2} \mid \mathcal{F}_{0}\right] \\
& +\Delta t^{2} \mathbb{E}\left[\sup _{0 \leq m \leq n}\left|\sum_{k=0}^{m-2}\left(L^{j} a\left(t_{k}, U_{k}\right)-L^{j} a\left(t_{k}, Y_{k}\right)\right) \Delta W_{k}^{j}\right|^{2} \mid \mathcal{F}_{0}\right] \\
& \left.+\Delta t^{2} \mathbb{E}\left[\sup _{0 \leq m \leq n}\left|\sum_{k=0}^{m-2}\left(L^{j 1} L^{j 2} a\left(t_{k}, U_{k}\right)-L^{j_{1}} L^{j_{2}} a\left(t_{k}, Y_{k}\right)\right) I_{\left(j_{1}, j_{2}\right)}\right|^{2} \mid \mathcal{F}_{0}\right]\right\} \begin{array}{c}
\text { Terms } \\
\text { from } \\
A_{k}
\end{array} \\
& +\sum_{\alpha \in \mathcal{A}_{2}^{*}} \mathbb{E}\left[\sup _{0 \leq m \leq n}\left|\sum_{k=0}^{m-2} I_{\alpha}\left[f_{\alpha}\left(t_{k}, U_{k}\right)-f_{\alpha}\left(t_{k}, Y_{k}\right)\right]_{t_{k}, t_{k+2}}\right|^{2} \mid \mathcal{F}_{0}\right] \mid \begin{array}{c}
\text { Terms } \\
\text { from } \\
B_{k}
\end{array} \\
& \left.\left.\left.+\sum_{\alpha \in \mathcal{A}_{2}^{*}} \mathbb{E}\left[\sup _{0 \leq m \leq n}\left|\sum_{k=0}^{m-2} I_{\alpha}\left[f_{\alpha}\left(t_{k}, U_{k}\right)-f_{\alpha}\left(t_{k}, Y_{k}\right)\right]_{t_{k}, t_{k+1}}\right|^{2} \mid \mathcal{F}_{0}\right]\right\}\left.^{m-2}\right|^{2} \mid \mathcal{F}_{0}\right]\right) . \\
& +\mathbb{E}\left[\sup _{0 \leq m \leq n}\left|\sum_{k=0}^{m-2} R_{k}\right|^{2}\right] .
\end{aligned}
$$


We go term-by-term:

$$
\begin{aligned}
& \Delta t^{2} \mathbb{E}\left[\sup _{0 \leq m \leq n}\left|\sum_{k=0}^{m-2} a\left(t_{k+1}, U_{k+1}\right)-a\left(t_{k+1}, Y_{k+1}\right)\right|^{2} \mid \mathcal{F}_{0}\right] \\
& \leq \Delta t^{2} n \mathbb{E}\left[\sup _{0 \leq m \leq n} \sum_{k=0}^{m-2}\left|a\left(t_{k+1}, U_{k+1}\right)-a\left(t_{k+1}, Y_{k+1}\right)\right|^{2} \mid \mathcal{F}_{0}\right] \\
& \leq T \Delta t \mathbb{E}\left[\sum_{k=0}^{n-2}\left|a\left(t_{k+1}, U_{k+1}\right)-a\left(t_{k+1}, Y_{k+1}\right)\right|^{2} \mid \mathcal{F}_{0}\right] \\
& \leq C \Delta t \sum_{k=0}^{n-2} \mathbb{E}\left[\left|U_{k+1}-Y_{k+1}\right|^{2} \mid \mathcal{F}_{0}\right] \\
& \leq C \Delta t \sum_{k=0}^{n-1} Z_{k} .
\end{aligned}
$$

Similarly,

$$
\Delta t^{2} \mathbb{E}\left[\sup _{0 \leq m \leq n}\left|\sum_{k=0}^{m-2} a\left(t_{k}, U_{k}\right)-a\left(t_{k}, Y_{k}\right)\right|^{2} \mid \mathcal{F}_{0}\right] \leq C \Delta t \sum_{k=0}^{n-1} Z_{k} .
$$

Next, we consider the terms from $A_{k}$. From Lemma 2.1 with $\alpha=(j)$,

$$
\begin{aligned}
& \Delta t^{2} \mathbb{E}\left[\sup _{0 \leq m \leq n}\left|\sum_{k=0}^{m-2}\left(L^{j} a\left(t_{k}, U_{k}\right)-L^{j} a\left(t_{k}, Y_{k}\right)\right) \Delta W_{t_{k}, t_{k+1}}^{j}\right|^{2} \mid \mathcal{F}_{0}\right] \\
& \leq C \Delta t \sum_{k=0}^{n-2} Z_{k},
\end{aligned}
$$

and from Lemma 2.1 with $\alpha=\left(j_{1}, j_{2}\right)$,

$$
\begin{aligned}
\Delta t^{2} \mathbb{E}\left[\sup _{0 \leq m \leq n}\left|\sum_{k=0}^{m-2}\left(L^{j 1} L^{j 2} a\left(t_{k}, U_{k}\right)-L^{j_{1}} L^{j_{2}} a\left(t_{k}, Y_{k}\right)\right) I_{\left(j_{1}, j_{2}\right) t_{k}, t_{k+1}}\right|^{2} \mid \mathcal{F}_{0}\right] \\
\leq C \Delta t \sum_{k=0}^{n-1} Z_{k} .
\end{aligned}
$$

Now, we consider the terms from $B_{k}$. For $\alpha \in \mathcal{A}_{2}^{*}$ (i.e., $\alpha \in \mathcal{A}_{2}, \ell(\alpha) \neq n(\alpha)$ ),

$$
\mathbb{E}\left[\sup _{0 \leq m \leq n}\left|\sum_{k=0}^{m-2} I_{\alpha}\left[f_{\alpha}\left(t_{k}, U_{k}\right)-f_{\alpha}\left(t_{k}, Y_{k}\right)\right]_{t_{k}, t_{k+1}}\right|^{2} \mid \mathcal{F}_{0}\right] \leq C \Delta t \sum_{k=0}^{n-1} Z_{k} .
$$

The other terms from $B_{k}$ are similar. This leaves only the terms from $R_{k}$. From Lemma 2.2 with $\alpha=(0)$,

$$
\begin{aligned}
& \Delta t^{2} \mathbb{E}\left[\sup _{0 \leq m \leq n}\left|\sum_{k=0}^{m-2} L^{0} L^{0} a\left(t_{n}, U_{n}\right) \Delta t^{2}\right|^{2} \mid \mathcal{F}_{0}\right] \\
& \leq \Delta t^{4} \mathbb{E}\left[\sup _{0 \leq m \leq n}\left|\sum_{k=0}^{n-2} L^{0} L^{0} a\left(t_{n}, U_{n}\right)\right|^{2} \mid \mathcal{F}_{0}\right] \\
& \leq C \Delta t^{4}\left(1+\left|U_{0}\right|^{2}\right) .
\end{aligned}
$$


For $\alpha=(0, j),(j, 0),\left(j_{1}, j_{2}, j_{3}\right)$, by Lemma 2.2

$$
\begin{gathered}
\Delta t^{2} \mathbb{E}\left[\sup _{0 \leq m \leq n}\left|\sum_{k=0}^{m-2} I_{\alpha}\left[a_{\alpha}\left(t_{k}, U_{k}\right)\right]_{t_{k}, t_{k+1}}\right|^{2} \mid \mathcal{F}_{0}\right] \\
\leq C \Delta t^{4}\left(1+\left|U_{0}\right|^{2}\right), \text { since } \ell(\alpha)+n(\alpha)=3 .
\end{gathered}
$$

If $\alpha \in \mathcal{B}(\mathcal{A} \gamma)$ (here $\gamma=1.5$ or 2.0), we have by Lemma 2.2,

$$
\mathbb{E}\left[\sup _{0 \leq m \leq n}\left|\sum_{k=0}^{m-2} I_{\alpha}[\alpha(\cdot, U .)]_{t_{k}, t_{k+1}}\right|^{2} \mid \mathcal{F}_{0}\right] \leq C\left(1+\left|U_{0}\right|^{2}\right) \Delta t^{2 \gamma} .
$$

Therefore, we have

$$
\mathbb{E}\left[\sup _{0 \leq m \leq n}\left|\sum_{k=0}^{m-2} R_{k}\right|^{2} \mid \mathcal{F}_{0}\right] \leq C\left(1+\left|U_{0}\right|^{2}\right) \Delta t^{4} .
$$

So, overall, we see that

$$
Z_{n} \leq C\left[Z_{1}+\left(1+\left|U_{0}\right|^{2}\right) \Delta t^{4}+\Delta t \sum_{k=0}^{n-1} Z_{k}\right]
$$

The result then follows from Lemma 2.3.

Remark 3.1. If we truncate $A_{n}$ and $B_{n}$ to

$$
A_{n}(t, x)=L^{j} a(t, x) \Delta W^{j}
$$

and

$$
\begin{aligned}
B_{n}(t, x)= & b^{j}(t, x) \Delta W^{j}+L^{0} b^{j}(t, x) I_{(0, j)}+L^{j} a(t, x) I_{(j, 0)} \\
& +L^{j_{1}} b^{j_{2}}(t, x) I_{\left(j_{1}, j_{2}\right)}+L^{j_{1}} L^{j_{2}} b^{j_{3}}(t, x) I_{\left(j_{1}, j_{2}, j_{3}\right)},
\end{aligned}
$$

the same proof will show that the convergence is now to order $\Delta t^{\frac{3}{2}}$. We note that although the order $\Delta t^{2} \mathrm{SAB}$ scheme seems to have no obvious advantages over the standard $\Delta t^{2}$ strong one-step explicit scheme (as in [6]), the order $\Delta t^{\frac{3}{2}}$ SAB scheme does have an advantage over the order $\Delta t^{\frac{3}{2}}$ strong one-step explicit scheme in that the former lacks the terms involving the second derivative of $a$ which are present in the latter.

Remark 3.2. It can be shown that the scheme

$$
\begin{aligned}
Y_{n+2}= & Y_{n+1}+\left[\frac{3}{2} a\left(t_{n+1}, Y_{n+1}\right)-\frac{1}{2} a\left(t_{n}, Y_{n}\right)\right] \Delta t \\
& -\frac{3}{2} \Delta t A_{n}\left(t_{n}, Y_{n}\right)+B_{n}\left(t_{n}, Y_{n}\right)
\end{aligned}
$$

in which

$$
A_{n}(t, x)=L^{j} a(t, x) \Delta W^{j},
$$

where the random intervals are from time $t_{n}$ to $t_{n+1}$, and

$$
\begin{aligned}
B_{n}(t, x)= & b^{j}(t, x) \Delta W^{j}+L^{0} b^{j}(t, x) I_{(0, j)}+L^{j} a(t, x) I_{(j, 0)} \\
& +L^{j_{1}} b^{j_{2}}(t, x) I_{\left(j_{1}, j_{2}\right)},
\end{aligned}
$$


where the random intervals are those from time $t_{n}$ to $t_{n+2}$ minus those from time $t_{n}$ to $t_{n+1}$, converges to the Itô solution in the weak sense to order 2 . As can be seen, this scheme is considerably simpler than the strong scheme, and it avoids the difficulties with generating the higher-order moments that the strong scheme has.

4. A stochastic "implicit leapfrog" scheme. The barotropic vorticity model supplied to us by Cecile Penland and Prashant Sardeshmukh of the National Oceanic and Atmospheric Administration in Boulder, Colorado (see [11]), takes the form

$$
\frac{\partial \zeta}{\partial t}=-\nabla \cdot(v \zeta)+S-r \xi-\kappa \nabla^{4} \xi
$$

where $\zeta=\nabla^{2} \psi+f=\xi+f$ and $v=\hat{k} \times \nabla \psi$. Here, $\zeta$ is the total vorticity, $v$ is the velocity vector, $f$ is the Coriolis term, $S$ is a (deterministic) forcing, $r$ and $\kappa$ are constants, and $\xi$ is the local vorticity.

The numerical scheme they provided for this uses spherical harmonics, and, writing $F$ for $-\nabla \cdot(v \zeta)$, the equation becomes

$$
\frac{d}{d t} \zeta_{n}^{m}=F_{n}^{m}+S_{n}^{m}-r \xi_{n}^{m}-\kappa\left[\frac{n(n+1)}{a^{2}}\right]^{2} \zeta_{n}^{m} .
$$

Then the scheme has two steps. First, a leapfrog step,

$$
\tilde{\zeta}_{n}^{m}(t+\Delta t)=\zeta_{n}^{m}(t-\Delta t)+2 \Delta t\left[F_{n}^{m}(t)+S_{n}^{m}(t)\right],
$$

followed by an implicit step,

$$
\zeta_{n}^{m}(t+\Delta t)=\frac{\tilde{\zeta}_{n}^{m}(t+\Delta t)}{1+2 \Delta t\left[r+\kappa\left[\frac{n(n+1)}{a^{2}}\right]^{2}\right]} .
$$

If we simplify notation and write $a_{1}$ for $F+S$ and $a_{2}$ for $-r \xi-\kappa \nabla^{4} \xi$, we see that this is just an "implicit leapfrog" scheme

$$
\left\{\begin{array}{l}
\tilde{Y}(t+\Delta t)=Y(t-\Delta t)+2 \Delta t a_{1}(t, Y(t)) \\
Y(t+\Delta t)=\tilde{Y}(t+\Delta t)+2 \Delta t a_{2}(t+\Delta t, Y(t+\Delta t))
\end{array}\right.
$$

for the equation

$$
d U(t)=\left[a_{1}(t, U(t))+a_{2}(t, U(t))\right] d t .
$$

Therefore, we consider a stochastic differential equation of the form

$$
d U_{t}=\left(a_{1}\left(t, U_{t}\right)+a_{2}\left(t, U_{t}\right)\right) d t+b\left(t, U_{t}\right) d W_{t} .
$$

Note that we have simply added a general diffusion term to the deterministic differential equation (4.6).

We will consider the scheme

$$
\left\{\begin{array}{l}
\tilde{Y}_{n+2}=Y_{n}+2 a_{1}\left(t_{n+1}, Y_{n+1}\right) \Delta t+M_{n}\left(Y_{n}\right)+M_{n+1}\left(Y_{n+1}\right), \\
Y_{n+2}=\tilde{Y}_{n+2}+2 a_{2}\left(t_{n+2}, Y_{n+2}\right) \Delta t,
\end{array}\right.
$$

where

$$
M_{n}(y)=b\left(t_{n}, y\right) \Delta W_{n}+b b^{\prime}\left(t_{n}, y\right) I_{(1,1), n} .
$$


THEOREM 4.1. Suppose that the coefficient functions $f_{\alpha}$ satisfy

$$
\left|f_{\alpha}(t, x)-f_{\alpha}(t, y)\right| \leq K|x-y|
$$

for all $\alpha \in \mathcal{A}_{1.0}, t \in[0, T]$, and $x, y \in \mathbb{R}^{d}$;

$$
f_{-\alpha} \in C^{1,2} \quad \text { and } \quad f_{\alpha} \in \mathcal{H}_{\alpha}
$$

for all $\alpha \in \mathcal{A}_{1.0} \cup \mathcal{B}\left(\mathcal{A}_{1.0}\right)$; and

$$
\left|f_{\alpha}(t, x)\right| \leq K(1+|x|)
$$

for all $\alpha \in \mathcal{A}_{1.0} \cup \mathcal{B}\left(\mathcal{A}_{1.0}\right), t \in[0, T]$, and $x \in \mathbb{R}^{d}$. Choose $\Delta t \leq 1$ and set $N=T / \Delta t$, and define $t_{n}=n \Delta t$ for $n=1, \ldots, N$. Suppose that some appropriate numerical scheme is used to generate $Y_{1}$ such that $\mathbb{E}\left[\left|U_{t_{1}}-Y_{1}\right|^{2} \mid \mathcal{F}_{0}\right]^{\frac{1}{2}} \leq C \Delta t$. Then

$$
\mathbb{E}\left[\sup _{0 \leq n \leq N}\left|U_{t_{n}}-Y_{n}\right|^{2} \mid \mathcal{F}_{0}\right]^{\frac{1}{2}} \leq C \Delta t .
$$

Proof. We note first that, by Itô's formula (i.e., the Taylor expansion with $\gamma=$ 0.0 ), the solution $U$ to (4.7) satisfies the following equations (where for notational simplicity we have written $U_{n}$ for $\left.U_{t_{n}}\right)$ :

$$
\begin{aligned}
& a_{1}\left(t_{n+1}, U_{n+1}\right)=a_{1}\left(t_{n}, U_{n}\right)+R_{0.0}^{\Delta t, a_{1}}\left(t_{n}\right) ; \\
& a_{2}\left(t_{n+2}, U_{n+2}\right)=a_{2}\left(t_{n}, U_{n}\right)+R_{0.0}^{2 \Delta t, a_{2}}\left(t_{n}\right) ; \\
& a_{2}\left(t_{n+2}, U_{n+2}\right)=a_{2}\left(t_{n+1}, U_{n+1}\right)+R_{0.0}^{\Delta t, a_{2}}\left(t_{n+1}\right) .
\end{aligned}
$$

Therefore we have

$$
\begin{aligned}
U_{n+2}= & U_{n}+\left(U_{n+2}-U_{n+1}\right)+\left(U_{n+1}-U_{n}\right) \\
= & U_{n}+\left[b\left(t_{n+1}, U_{n+1}\right) \Delta W_{n+1}+a_{1}\left(t_{n+1}, U_{n+1}\right) \Delta t\right. \\
& +a_{2}\left(t_{n+1}, U_{n+1}\right) \Delta t+b b^{\prime}\left(t_{n+1}, U_{n+1}\right) I_{(1,1), n+1} \\
& \left.+R_{1.0}^{\Delta t}\left(t_{n+1}\right)\right]+\left[b\left(t_{n}, U_{n}\right) \Delta W_{n}+a_{1}\left(t_{n}, U_{n}\right) \Delta t\right. \\
& \left.+a_{2}\left(t_{n}, U_{n}\right) \Delta t+b b^{\prime}\left(t_{n}, U_{n}\right) I_{(1,1), n}+R_{1.0}^{\Delta t}\left(t_{n}\right)\right] .
\end{aligned}
$$

After substituting (4.14) into this, we see that

$$
\begin{aligned}
U_{n+2}= & {\left[U_{n}+2 a_{1}\left(t_{n+1}, U_{n+1}\right) \Delta t+M_{n}\left(U_{n}\right)+M_{n+1}\left(U_{n+1}\right)\right] } \\
& +2 a_{2}\left(t_{n+2}, U_{n+2}\right) \Delta t+R_{n}
\end{aligned}
$$

here

$$
\begin{aligned}
R_{n}= & R_{1.0}^{\Delta t}\left(t_{n}\right)+R_{1.0}^{\Delta t}\left(t_{n+1}\right)-\Delta t\left[R_{0.0}^{\Delta t, a_{1}}\left(t_{n}\right)\right. \\
& \left.+R_{0.0}^{2 \Delta t, a_{2}}\left(t_{n}\right)+R_{0.0}^{\Delta t, a_{2}}\left(t_{n+1}\right)\right] .
\end{aligned}
$$

If we iterate (4.16), we arrive at

$$
\begin{aligned}
U_{n}= & U_{n^{*}}+2 \Delta t\left[\sum_{k=1}^{[n / 2]} a_{1}\left(t_{2 k-1+n^{*}}, U_{2 k-1+n^{*}}\right)+\sum_{k=1}^{[n / 2]} a_{2}\left(t_{2 k+n^{*}}, U_{2 k+n^{*}}\right)\right] \\
& +\sum_{k=n^{*}}^{n-1} M_{n}\left(U_{n}\right)+\sum_{k=0}^{[n / 2]} R_{2 k-2+n^{*}} ;
\end{aligned}
$$

here $n^{*}$ is 0 if $n$ is even and 1 if $n$ is odd. 
Similarly, we have for $Y$

$$
\begin{aligned}
Y_{n}= & Y_{n^{*}}+2 \Delta t\left[\sum_{k=1}^{[n / 2]} a_{1}\left(t_{2 k-1+n^{*}}, Y_{2 k-1+n^{*}}\right)+\sum_{k=1}^{[n / 2]} a_{2}\left(t_{2 k+n^{*}}, Y_{2 k+n^{*}}\right)\right] \\
& +\sum_{k=n^{*}}^{n-1} M_{n}\left(Y_{n}\right) .
\end{aligned}
$$

Let us set $Z_{n}=\mathbb{E}\left[\sup _{0 \leq m \leq n}\left|U_{m}-Y_{m}\right|^{2} \mid \mathcal{F}_{0}\right]$. Then, by subtracting (4.19) from (4.18) and then squaring and taking expectations, we find

$$
\begin{aligned}
Z_{n} \leq & C \mathbb{E}\left[\sup _{0 \leq m \leq n} Z_{m^{*}}+\Delta t^{2} A_{1, m}^{2}+\Delta t^{2} A_{2, m}^{2}\right. \\
& \left.+\left(\sum_{k=m^{*}}^{m-1} M_{k}\left(U_{k}\right)-M_{k}\left(Y_{k}\right)\right)^{2}+\left(\sum_{k=0}^{[m / 2]} R_{2 k-2+m^{*}}\right)^{2} \mid \mathcal{F}_{0}\right] .
\end{aligned}
$$

In (4.20),

$$
A_{1, n}=\sum_{k=1}^{[n / 2]} a_{1}\left(t_{2 k-1+n^{*}}, U_{2 k-1+n^{*}}\right)-a_{1}\left(t_{2 k-1+n^{*}}, Y_{2 k-1+n^{*}}\right)
$$

and

$$
A_{2, n}=\sum_{k=1}^{[n / 2]} a_{2}\left(t_{2 k+n^{*}}, U_{2 k+n^{*}}\right)-a_{2}\left(t_{2 k+n^{*}}, Y_{2 k+n^{*}}\right) .
$$

We then have the following estimates (where we omit the dependence on $t$ when it is clear):

$$
\begin{aligned}
\Delta t^{2} \mathbb{E}\left[\sup _{0 \leq m \leq n} A_{1, m}^{2}\right] & \leq \Delta t^{2} \mathbb{E}\left[\sup _{0 \leq m \leq n}\left[\frac{m}{2}\right] \sum_{k=1}^{[m / 2]}\left[a_{1}\left(U_{2 k-1+n^{*}}\right)-a_{1}\left(Y_{2 k-1+n^{*}}\right)\right]^{2}\right] \\
& \leq K \Delta t^{2} n \mathbb{E}\left[\sum_{k=1}^{n}\left(U_{k}-Y_{k}\right)^{2}\right] \leq K T \Delta t \sum_{k=1}^{n} Z_{k}
\end{aligned}
$$

Similarly,

$$
\Delta t^{2} \mathbb{E}\left[\sup _{0 \leq m \leq n} A_{2, n}^{2}\right] \leq K T \Delta t \sum_{k=1}^{n} Z_{k}
$$

There are two terms in $M_{k}$. For the first one, from Lemma 2.1, with $\alpha=(1)$, we obtain

$$
\mathbb{E}\left[\sup _{0 \leq m \leq n}\left|\sum_{k=m^{*}}^{m-1}\left[b\left(U_{k}\right)-b\left(Y_{k}\right)\right] \Delta W_{k}\right|^{2}\right] \leq C \Delta t \sum_{k=0}^{n} Z_{k} .
$$

The second term is similar, with $\alpha=(1,1)$. 
Finally, we show a representative term from the remainder $R$. From Lemma 2.2, with $\alpha=(1,0)$,

$$
\mathbb{E}\left[\sup _{0 \leq m \leq n}\left|\sum_{k=0}^{m-1} \int_{t_{k}}^{t_{k+1}} \int_{t_{k}}^{s} L^{0} b\left(U_{r}\right) d r d W_{s}\right|^{2}\right] \leq C \Delta t^{2}\left(1+U_{0}^{2}\right) .
$$

The remaining terms from $R$ give similar bounds.

Taking all of these estimates into account, we have the inequality

$$
Z_{n} \leq K\left[Z_{1}+\Delta t \sum_{k=0}^{n} Z_{k}+\Delta t^{2}\right]
$$

Therefore, from Lemma 2.3, we see that

$$
\mathbb{E}\left[\sup _{o \leq n \leq N}\left|U_{n}-Y_{n}\right|^{2} \mid \mathcal{F}_{0}\right]^{\frac{1}{2}}=O(\Delta t)
$$

Remark 4.1. It is possible to show that the scheme

$$
\left\{\begin{aligned}
\tilde{Y}_{n+2}= & Y_{n}+2 a_{1}\left(t_{n+1}, Y_{n+1}\right) \Delta t+b\left(t_{n}, Y_{n}\right) \Delta W_{n} \\
& +b\left(t_{n+1}, Y_{n+1}\right) \Delta W_{n+1} \\
Y_{n+2}= & \tilde{Y}_{n+2}+2 a_{2}\left(t_{n+2}, Y_{n+2}\right) \Delta t
\end{aligned}\right.
$$

converges to the Itô solution in the weak sense to order 1. Again, it can be seen that the weak scheme is simpler than the strong scheme to the same order. However, we also note that the weak order 1 Stratonovich scheme

$$
\left\{\begin{aligned}
\tilde{Y}_{n+2}= & Y_{n}+2 a_{1}\left(t_{n+1}, Y_{n+1}\right) \Delta t+b b^{\prime}\left(t_{n+1}, Y_{n+1}\right)\left(\Delta W_{n+1}\right)^{2} \\
& +b\left(t_{n}, Y_{n}\right) \Delta W_{n}+b\left(t_{n+1}, Y_{n+1}\right) \Delta W_{n+1} \\
Y_{n+2}= & \tilde{Y}_{n+2}+2 a_{2}\left(t_{n+2}, Y_{n+2}\right) \Delta t
\end{aligned}\right.
$$

is not appreciably simpler than the strong order 1 Stratonovich scheme

$$
\left\{\begin{array}{l}
\tilde{Y}_{n+2}=Y_{n}+2 a_{1}\left(t_{n+1}, Y_{n+1}\right) \Delta t+M_{n}\left(Y_{n}\right)+M_{n+1}\left(Y_{n+1}\right), \\
Y_{n+2}=\tilde{Y}_{n+2}+2 a_{2}\left(t_{n+2}, Y_{n+2}\right) \Delta t,
\end{array}\right.
$$

where

$$
M_{n}(y)=b\left(t_{n}, y\right) \Delta W_{n}+\frac{1}{2} b b^{\prime}\left(t_{n}, y\right)\left(\Delta W_{n}\right)^{2} .
$$

5. Discretization of spatial derivatives by finite differences. It sometimes happens in applications that the functions $a$ and $b$ may only be known empirically (i.e., in tables) rather than analytically. In such cases, analytic derivatives of these functions can be difficult to obtain. It is therefore useful to replace these derivatives by discrete approximations. As a first example, consider this modification of Mil'shtein's scheme:

$$
\begin{aligned}
\hat{Y}_{n+1}^{k}= & \hat{Y}_{n}^{k}+\sum_{j=1}^{d} b^{k, j}\left(\hat{Y}_{n}\right) \Delta W_{n}^{j}+a^{k}\left(\hat{Y}_{n}\right) \Delta t \\
& +\sum_{j_{1}, j_{2}, \ell=1}^{d} \frac{1}{\Delta x} b^{\ell, j_{1}}\left(\hat{Y}_{n}\right)\left(b^{k, j_{2}}\left(\hat{Y}_{n}+\Delta x e^{\ell}\right)-b^{k, j_{2}}\left(\hat{Y}_{n}\right)\right) I_{\left(j_{1}, j_{2}\right), n},
\end{aligned}
$$


where $e^{\ell}$ is the vector $(0, \ldots, 0,1,0, \ldots, 0)$, with 1 in the $\ell$ th position, and we have chosen $\Delta x>0$. We have also suppressed the dependence of $a$ and $b$ on time to simplify notation.

We then have the following theorem.

THEOREM 5.1. Suppose that $a$ and $b$ have the regularity required for Mil'shtein's scheme to converge to the solution $U$ to order $\Delta t$. Then

$$
\mathbb{E}\left[\sup _{0 \leq n \leq N}\left|U_{n}-\hat{Y}_{n}\right|^{2}\right]^{\frac{1}{2}}=O\left(\max \left\{\Delta t, \Delta x \Delta t^{\frac{1}{2}}\right\}\right) .
$$

Note that if we want to maintain the order of convergence of Mil'shtein's scheme, we need that $\Delta x=O\left(\Delta t^{\frac{1}{2}}\right)$.

Proof. We denote Mil'shtein's scheme by $Y$ and recall that it satisfies

$$
\mathbb{E}\left[\sup _{0 \leq n \leq N}\left|Y_{n}-U_{n}\right|^{2}\right]^{\frac{1}{2}}=O(\Delta t) .
$$

First, we see that (using Einstein's summation convention on repeated indices)

$$
\begin{gathered}
\hat{Y}_{n+1}^{k}-Y_{n+1}^{k}=\hat{Y}_{n}^{k}-Y_{n}^{k}+\left(b^{k, j}\left(\hat{Y}_{n}\right)-b^{k, j}\left(Y_{n}\right)\right) \Delta W_{n}^{j}+\left(a^{k}\left(\hat{Y}_{n}\right)-a^{k}\left(Y_{n}\right)\right) \Delta t \\
+\left[b^{\ell, j_{1}}\left(\hat{Y}_{n}\right) \frac{1}{\Delta x}\left(b^{k, j_{2}}\left(\hat{Y}_{n}+\Delta x e^{\ell}\right)-b^{k, j_{2}}\left(\hat{Y}_{n}\right)\right)\right. \\
\left.\quad-b^{\ell, j_{1}}\left(Y_{n}\right) \frac{\partial b^{k, j_{2}}}{\partial x^{\ell}}\left(Y_{n}\right)\right] I_{\left(j_{1}, j_{2}\right), n} .
\end{gathered}
$$

Iterating this, we have

$$
\begin{gathered}
\hat{Y}_{n}^{k}-Y_{n}^{k}=\hat{Y}_{0}^{k}-Y_{0}^{k}+\sum_{i=0}^{n-1}\left(b^{k, j}\left(\hat{Y}_{i}\right)-b^{k, j}\left(Y_{i}\right)\right) \Delta W_{i}^{j}+\sum_{i=0}^{n-1}\left(a^{k}\left(\hat{Y}_{i}\right)-a^{k}\left(Y_{i}\right)\right) \Delta t \\
+\sum_{i=0}^{n-1}\left[b^{\ell, j_{1}}\left(\hat{Y}_{i}\right) \frac{1}{\Delta x}\left(b^{k, j_{2}}\left(\hat{Y}_{i}+\Delta x e^{\ell}\right)-b^{k, j_{2}}\left(\hat{Y}_{i}\right)\right)\right. \\
\left.-b^{\ell, j_{1}}\left(Y_{i}\right) \frac{\partial b^{k, j_{2}}}{\partial x^{\ell}}\left(Y_{i}\right)\right] I_{\left(j_{1}, j_{2}\right), i} .
\end{gathered}
$$

Set

$$
Z_{n}=\mathbb{E}\left[\sup _{0 \leq m \leq n}\left|\hat{Y}_{n}-Y_{n}\right|^{2}\right] .
$$

We then have the estimates

$$
\begin{aligned}
& \mathbb{E}\left[\sup _{0 \leq m \leq n}\left|\sum_{i=0}^{m-1}\left(b^{k, j}\left(\hat{Y}_{i}\right)-b^{k, j}\left(Y_{i}\right)\right) \Delta W_{i}^{j}\right|^{2}\right] \\
& \leq 4 \mathbb{E}\left[\left|\sum_{i=0}^{n-1}\left(b^{k, j}\left(\hat{Y}_{i}\right)-b^{k, j}\left(Y_{i}\right)\right) \Delta W_{i}^{j}\right|^{2}\right] \\
& \leq 4 \mathbb{E}\left[\sum_{i=0}^{n-1}\left|b^{k, j}\left(\hat{Y}_{i}\right)-b^{k, j}\left(Y_{i}\right)\right|^{2} \Delta t\right] \\
& \leq C \Delta t \sum_{i=0}^{n-1} Z_{i},
\end{aligned}
$$


and

$$
\begin{aligned}
\mathbb{E}\left[\sup _{0 \leq m \leq n}\left|\sum_{i=0}^{m-1}\left(a^{k}\left(\hat{Y}_{i}\right)-a^{k}\left(Y_{i}\right)\right) \Delta t\right|^{2}\right] & \leq \Delta t^{2} n \mathbb{E}\left[\sum_{i=0}^{n-1}\left|a^{k}\left(\hat{Y}_{i}\right)-a^{k}\left(Y_{i}\right)\right|^{2}\right] \\
& \leq C \Delta t \sum_{i=0}^{n-1} Z_{i},
\end{aligned}
$$

and, finally,

$$
\begin{aligned}
\mathbb{E}\left[\sup _{0 \leq m \leq n} \mid \sum_{i=0}^{m-1}\left[b^{\ell, j_{1}}\left(\hat{Y}_{i}\right) \frac{1}{\Delta x}\left[b^{k, j_{2}}\left(\hat{Y}_{i}+\Delta x e^{\ell}\right)-b^{k, j_{2}}\left(\hat{Y}_{i}\right)\right]\right.\right. \\
\left.\left.\quad-b^{\ell, j_{1}}\left(Y_{i}\right) \frac{\partial b^{k, j_{2}}}{\partial x^{\ell}}\left(Y_{i}\right)\right]\left.I_{\left(j_{1}, j_{2}\right), i}\right|^{2}\right] \\
\leq C \Delta t^{2} \sum_{i=0}^{n-1} \mathbb{E}\left[b^{\ell, j_{1}}\left(\hat{Y}_{i}\right) \frac{1}{\Delta x}\left[b^{k, j_{2}}\left(\hat{Y}_{i}+\Delta x e^{\ell}\right)-b^{k, j_{2}}\left(\hat{Y}_{i}\right)\right]-b^{\ell, j_{1}}\left(Y_{i}\right) \frac{\partial b^{k, j_{2}}}{\partial x^{\ell}}\left(Y_{i}\right)\right]^{2} \\
\leq C \Delta t^{2} \sum_{i=0}^{n-1} \mathbb{E}\left[b^{\ell, j_{1}}\left(\hat{Y}_{i}\right) \frac{\partial b^{k, j_{2}}}{\partial x^{\ell}}\left(\hat{Y}_{i}\right)-b^{\ell, j_{1}}\left(Y_{i}\right) \frac{\partial b^{k, j_{2}}}{\partial x^{\ell}}\left(Y_{i}\right)\right]^{2} \\
\quad+C \Delta t^{2} \sum_{i=0}^{n-1} b^{\ell, j_{1}}\left(\hat{Y}_{i}\right)^{2}\left[\frac{1}{\Delta x}\left(b^{k, j_{2}}\left(\hat{Y}_{i}+\Delta x e^{\ell}\right)-b^{k, j_{2}}\left(\hat{Y}_{i}\right)\right)-\frac{\partial b^{k, j_{2}}}{\partial x^{\ell}}\left(\hat{Y}_{i}\right)\right]^{2} \\
\leq C \Delta t^{2} \sum_{i=0}^{n-1} Z_{i}+C \Delta t \Delta x^{2} .
\end{aligned}
$$

Therefore, altogether we have

$$
Z_{n} \leq C \Delta t \sum_{k=0}^{n-1} Z_{k}+C \Delta t \Delta x^{2}
$$

and an application of Lemma 2.3 implies that

$$
\mathbb{E}\left[\sup _{0 \leq n \leq N}\left|Y_{n}-\hat{Y}_{n}\right|^{2}\right]^{\frac{1}{2}}=O\left(\Delta x \Delta t^{\frac{1}{2}}\right)
$$

We can apply a similar idea to the SAB scheme. That is, if we replace $A_{n}$ and $B_{n}$ in the order $\Delta t^{\frac{3}{2}}$ scheme (see Remark 3.1) by, for instance (with, again, Einstein's summation convention in effect),

$$
A_{n}(t, x)=b^{k j}(t, x) \frac{1}{\Delta x}\left[a\left(t, x+\Delta x e^{k}\right)-a(t, x)\right] \Delta W^{j},
$$

where $e^{k}=(0, \ldots, 0,1,0, \ldots, 0)$, with the 1 in the $k$ th position, and 


$$
\begin{aligned}
B_{n}(t, x)= & b^{j}(t, x) \Delta W^{j}+\frac{1}{\Delta t}\left[b^{j}(t+\Delta t, x)-b^{j}(t, x)\right] I_{(0, j), n} \\
+ & a^{k}(t, x) \frac{1}{\Delta x}\left[b^{j}\left(t, x+\Delta x e^{k}\right)-b^{j}(t, x)\right] I_{(0, j), n} \\
+ & b^{k i}(t, x) b^{\ell i}(t, x) \frac{1}{8 \Delta x^{2}}\left[b^{j}\left(t, x+\Delta x\left(e^{i}+e^{\ell}\right)\right)-b^{j}\left(t, x+\Delta x\left(e^{i}-e^{\ell}\right)\right)\right. \\
& \left.-b^{j}\left(t, x+\Delta x\left(e^{\ell}-e^{i}\right)\right)+b^{j}\left(t, x-\Delta x\left(e^{i}+e^{\ell}\right)\right)\right] I_{(0, j), n} \\
+ & b^{k j_{1}}(t, x) \frac{1}{2 \Delta x}\left[b^{j_{2}}\left(t, x+\Delta x e^{k}\right)-b^{j_{2}}\left(t, x-\Delta x e^{k}\right)\right] I_{\left(j_{1}, j_{2}\right), n} \\
+ & b^{k j}(t, x) \frac{1}{\Delta x}\left[a\left(t, x+\Delta x e^{k}\right)-a(t, x)\right] I_{(j, 0), n} \\
+ & b^{k_{1} j_{1}}(t, x) \frac{1}{4 \Delta x^{2}}\left[b^{k_{2} j_{2}}\left(t, x+\Delta x e^{k_{1}}\right)-b^{k_{2} j_{2}}\left(t-x+\Delta x e^{k_{1}}\right)\right] \\
& {\left[b^{j_{3}}\left(t, x+\Delta x e^{k_{2}}\right)-b^{j_{3}}\left(t, x-\Delta x e^{k_{1}}\right)\right] I_{\left(j_{1}, j_{2}, j_{3}\right), n} } \\
+ & b^{k_{1} j_{1}}(t, x) b^{k_{2}, j_{2}}(t, x) \frac{1}{4 \Delta x^{2}} \\
& {\left[b^{j_{3}}\left(t, x+\Delta x\left(e^{k_{1}}+e^{k_{2}}\right)\right)-b^{j_{3}}\left(t, x+\Delta x\left(e^{k_{1}}-e^{k_{2}}\right)\right)\right.} \\
& \left.\quad-b^{j_{3}}\left(t, x+\Delta x\left(e^{k_{2}}-e^{k_{1}}\right)\right)+b^{j_{3}}\left(t, x-\Delta x\left(e^{k_{1}}+e^{k_{2}}\right)\right)\right] I_{\left(j_{1}, j_{2}, j_{3}\right), n},
\end{aligned}
$$

we could then prove that this scheme converges to order $\max \left\{\Delta t^{\frac{3}{2}}, \Delta t^{\frac{1}{2}} \Delta x\right\}$ in a similar fashion.

6. Numerical simulation. The object of this section is to test numerically the accuracy of the scheme of section 3 and compare it to the theoretical result above (i.e., $O\left(\Delta t^{2}\right)$ accuracy) and to the accuracy of the Euler and Mil'shtein schemes (respectively, $O\left(\Delta t^{\frac{1}{2}}\right)$ and $O(\Delta t)$ ). All the numerical results below are consistent with the theoretical ones.

We consider the following equation:

$$
d X_{t}=\beta^{2} \sinh X_{t} \cosh ^{2} X_{t} d t+\beta \cosh ^{2} X_{t} d W_{t},
$$

with $\beta=\frac{1}{10}$ and $X_{0}=\frac{1}{2}$. This has the exact solution

$$
X_{t}=\operatorname{arctanh}\left(\beta W_{t}+\tanh X_{0}\right),
$$

respectively. This can be easily verified using Itô's formula and is just one of many possible examples listed in [6].

We computed approximate solutions $Y_{n}$ using the Euler and Mil'shtein schemes and the SAB scheme from section 3 . Then we computed the following error:

$$
e=\sqrt{\mathbb{E}\left(\sup _{0 \leq n \leq N}\left|X_{n}-Y_{n}\right|^{2}\right)} .
$$

To estimate the mean value needed, we used 500 sample trajectories.

In Figure 6.1, the order of each scheme is given by the slope of the corresponding line. So we can see that the orders are $\frac{1}{2}$ for Euler, 1 for Mil'shtein, and 2 for the $\mathrm{SAB}$ of section 3 . 


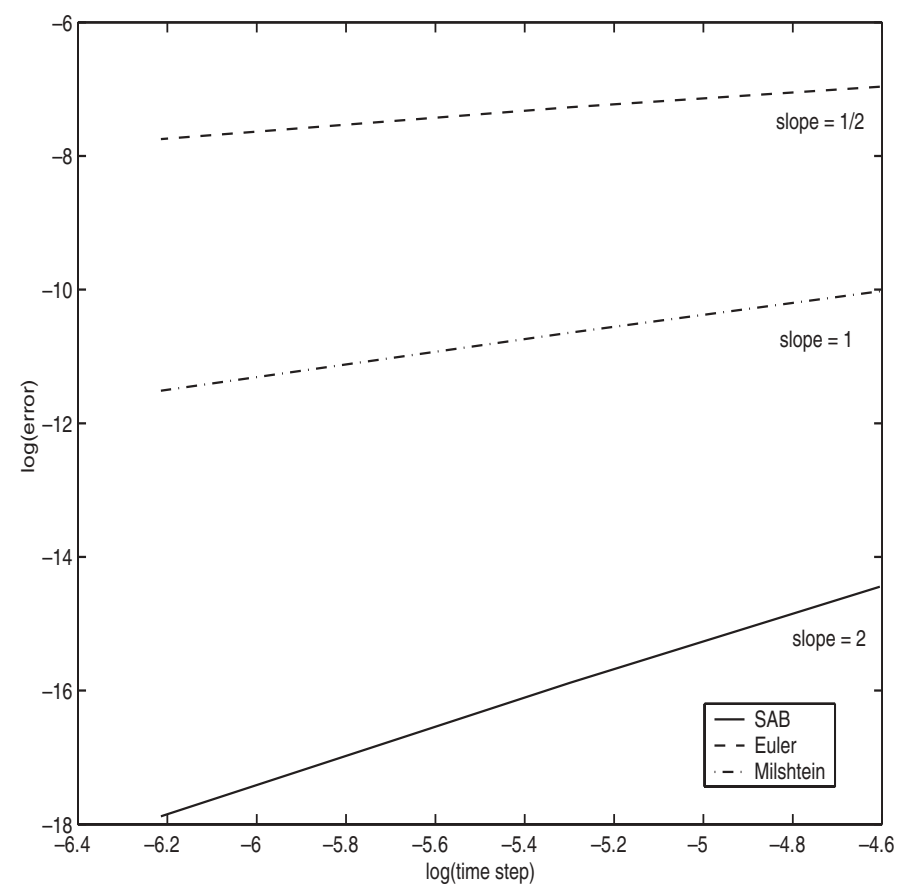

FIG. 6.1. Results obtained with the stochastic equation (6.1).

Note that for the SAB scheme, the stochastic integral $I_{(0,1,1)}$ (which is difficult to generate) was approximated by a normal law. The results tend to show that this does not affect the accuracy (at least in these two cases). We will try to improve this point, which seems to raise interesting probabilistic questions, as already mentioned in the introduction.

Acknowledgments. The authors are very grateful to Cecile Penland for bringing these issues to their attention, and they acknowledge very useful discussions with her and with Prashant Sardeshmukh. They are also indebted to Arnaud Debussche for several improvements on an earlier draft, and to Sylvain Faure, who provided the numerical simulations of section 6 .

\section{REFERENCES}

[1] V. Bally and D. TAlay, The law of the Euler scheme for stochastic differential equations, I. Convergence rate of the distribution function, Probab. Theory Related Fields, 104 (1996), pp. 43-60.

[2] V. Bally And D. Talay, The law of the Euler scheme for stochastic differential equations, II. Convergence rate of the density, Monte Carlo Methods Appl., 2 (1996), pp. 93-128.

[3] B. D. Ewald, Numerical Methods for Stochastic Differential Equations in the Geosciences, Ph.D. dissertation, Indiana University, Bloomington, 2002.

[4] B. D. Ewald, C. Penland, And R. Temam, Accurate integration of stochastic climate models with application to El Niño, Monthly Weather Rev., 132 (2004), pp. 154-164.

[5] J. G. Gaines And T. J. Lyons, Random generation of stochastic area integrals, SIAM J. Appl. Math., 54 (1994), pp. 1132-1146.

[6] P. E. Kloeden and E. Platen, Numerical Solution of Stochastic Differential Equations, Appl. Math. 23, Springer-Verlag, Berlin, 1992. 
[7] P. E. Kloeden, E. Platen, and H. Schurz, Numerical Solution of SDE through Computer Experiments, Universitext, Springer-Verlag, Berlin, 1994.

[8] G. N. Mil'shtein, Approximate integration of stochastic differential equations, Theory Probab. Appl., 19 (1974), pp. 557-562.

[9] C. Penland and P. D. Sardeshmukh, The optimal growth of tropical sea surface temperatures, J. Climate, 8 (1995), pp. 1999-2024.

[10] W. RüMELIN, Numerical treatment of stochastic differential equations, SIAM J. Numer. Anal., 19 (1982), pp. 604-613.

[11] P. D. Sardeshmukh and B. J. Hoskins, The generation of global rotational flow by steady idealized tropical divergence, J. Atmospheric Sci., 45 (1988), pp. 1228-1251.

[12] D. TAlay, Simulation of stochastic differential equations, in Probabilistic Methods in Applied Physics, P. Krée and W. Wedig, eds., Springer-Verlag, Berlin, 1995, pp. 54-96. 